\title{
Circuit
}

Musiques contemporaines

\section{Léo-Pol Morin en concert, de Claudine Caron, Montréal, Leméac éditeur, 2013, 249 pages}

\section{Anne Marie Messier}

Volume 25, numéro 2, 2015

URI : https://id.erudit.org/iderudit/1032938ar

DOI : https://doi.org/10.7202/1032938ar

Aller au sommaire du numéro

Éditeur(s)

Les Presses de l’Université de Montréal

ISSN

1183-1693 (imprimé)

1488-9692 (numérique)

Découvrir la revue

Citer ce compte rendu

Messier, A. M. (2015). Compte rendu de [Léo-Pol Morin en concert, de Claudine Caron, Montréal, Leméac éditeur, 2013, 249 pages]. Circuit, 25(2), 86-89.

https://doi.org/10.7202/1032938ar

Ce document est protégé par la loi sur le droit d'auteur. L'utilisation des services d'Érudit (y compris la reproduction) est assujettie à sa politique d'utilisation que vous pouvez consulter en ligne.

https://apropos.erudit.org/fr/usagers/politique-dutilisation/
Cet article est diffusé et préservé par Érudit.

Érudit est un consortium interuniversitaire sans but lucratif composé de l’Université de Montréal, l’Université Laval et l’Université du Québec à Montréal. Il a pour mission la promotion et la valorisation de la recherche. https://www.erudit.org/fr/ 


\section{Léo-Pol Morin en concert,}

\section{de Claudine Caron}

Montréal, Leméac éditeur, 2013, 249 pages.

Compte rendu d'Anne Marie Messier

Léo-Pol Morin (1892-1941) est une juste incarnation de l'artiste québécois éclairé, impliqué dans sa communauté, ouvert à la création internationale. Cependant il s'est vu oublié après sa mort, emporté dans le grand coup de vent de la Révolution tranquille, dont les acteurs, pour simplifier, ont préféré penser que leurs avancées relevaient d'une génération spontanée. Pianiste, chroniqueur, critique musical, conférencier, enseignant, il s'est fait le champion de la musique moderne. Il est temps que des chercheurs réhabilitent sa carrière et, dans ce sens, l'ouvrage de Claudine Caron $^{1}$ est plus que bienvenu. L'auteure y expose sa thèse de doctorat sur les concerts de Morin entre 1910 et 1941, année de sa mort prématurée dans un accident de voiture. La matière est riche, car Morin a donné plus de 250 concerts en salle et prestations à la radio, dont plusieurs sous forme de «causerie », moitié conférence, moitié récital. Étoffant la stricte analyse détaillée des programmes de concert et de leur réception par la presse de l'époque, elle adjoint quelques éléments biographiques qui situent l'évolution de l'artiste, son entourage, son implication dans la vie musicale et intellectuelle de l'époque. Louvrage divise le parcours de Morin en quatre chapitres: les débuts du pianiste

Claudine Caron

Léo-Pol Morin en concert

de 1908 à 1918; à Paris, dans la liberté de l'exil de 1919 à 1925; l'Amérique, de 1925 à 1929; et Montréal, de 1930 à 1941 .

Les années de formation passent d'un apprentissage de la musique à la maison à la formation musicale à Québec auprès de l'organiste et compositeur Gustave Gagnon et de Henri Gagnon, son fils. C'est dans cette classe des Gagnon que Morin fait un premier concert public en 1910 avec des œuvres de Bach et Moszkowski. Dès 1911, il participe à la première édition du Prix d'Europe, sans succès. Mais après quelques leçons à Montréal auprès d'Arthur Letondal et de Guillaume Couture, il obtient le Prix d'Europe en 1912, en présentant un programme classique qui comprend aussi Les jardins sous la pluie, de Debussy, préfigurant ce qui sera au cœur de toute sa carrière. Il part étudier à Paris 
avec Raoul Pugno puis avec Ricardo Viñes, où il s'imprègne de la vie culturelle très active - par exemple, il assiste en mai 1913 à la création du Sacre du printemps. Introduit par le poète québécois Marcel Dugas, il rencontre de nombreux artistes, Jean Cocteau entre autres, et surtout Maurice Ravel, avec qui il se liera d'amitié. C'est avec Viñes que Morin développe son goût pour les pièces modernes défendues par son professeur : Ravel, Debussy, le Groupe des six, les Russes, les Espagnols, l'École de Vienne. Dès ses premiers concerts à Paris en 1913 et en 1914, cette influence s'exprime avec des pièces de Fauré, de Franck, de Ravel, de Debussy.

De retour au Canada à cause de la guerre, Morin poursuit sa carrière toujours en incluant des pièces modernes dans son répertoire de récital, seul ou en formation de musique de chambre (avec violon ou avec voix). En 1916, il commence à s'intéresser à la musique canadienne et présente des œuvres d'Arthur Letondal, de Léo Roy, de Georges-Émile Tanguay, d'Henri Gagnon et surtout de Rodolphe Mathieu, dont il sera un interprète privilégié. C'est à cette époque que Morin commence à écrire, en réaction aux commentaires négatifs sur la musique moderne de certains critiques. Il publie un Claude Debussy, musicien français, mais pour lequel il est accusé d'avoir plagié un livre de Louis Laloy publié à Paris. Claudine Caron met des phrases en parallèle et le constat est flagrant; cependant elle n'y voit pas une grande faute et même admire la manière dont Morin «ajoute subtilement ses propres idées» (p. 53). Nous ne sommes pas certains que les lecteurs la suivront là-dessus... D’ailleurs, à plusieurs reprises, la lecture est heurtée par des choix de mots, des commentaires qui laissent pantois. Par exemple, un critique écrit sur l'assistance d'un concert qu'«un grand public particulièrement intellectuel se pressait dans la salle » ou que l'auditoire qui débordait de la salle était «très chic». Elle dit que ces commentaires donnent à penser que l'auditoire était très restreint (p. 55). Nous avouons ne pas avoir saisi le lien établi.

Revenons au parcours de Morin; après avoir simplement mentionné qu'il commence à enseigner en 1915, l'auteure insiste avec raison sur l'implication de Morin dans la création de la revue artistique d'avant-garde Le Nigog, fondée en 1918 avec l'écrivain Robert de Roquebrune et l'architecte Fernand Préfontaine. Passons outre le fait que l'auteure considère comme exceptionnel qu'une revue sur l'art contienne seulement des articles sur les différentes formes d'art (p. 57); il reste que Le Nigog est assurément une entrée importante dans la modernité au Québec, en ce qu'elle rejette la morale religieuse et cherche à émanciper le milieu artistique québécois. Pour Morin, c’est l'occasion d'un plaidoyer incessant pour la musique moderne au Canada et il commence à s'inscrire dans son domaine comme un expert et un intellectuel exigeant, position qu'il gardera tout au long de sa vie. Mais son sentiment d'isolement le pousse cependant à retourner en France pour y poursuivre sa carrière, ce qu'il fait après deux concerts d'adieu à Montréal et à Québec, où il joue essentiellement des œuvres modernes: du Debussy et du Ravel, comme à son habitude, mais aussi cinq œuvres canadiennes dont une première de lui-même, œuvre par ailleurs perdue.

De 1919, jusqu'à 1931, Morin sera résident de Paris, malgré de fréquents retours pour des concerts et pour d'autres activités musicales. Dès son arrivée, il reprend ses concerts et présente de nombreuses œuvres canadiennes, tout en reprenant des œuvres françaises de Debussy et de Ravel, mais aussi d'autres compositeurs, ce qui contribue à sa renommée d'interprète audacieux. En 1922, avec un nouvel imprésario, Bernard Laberge, il commence des années de tournées, seul ou comme accompagnateur. Il persiste dans ses programmations de modernes. Par exemple, dans une formule innovatrice sans applaudissements entre les pièces, il enchaîne des œuvres courtes: Grovlez, 
Fauré, Mathieu, Tansman, Poulenc, Honegger, Auric, Roland-Manuel, Roussel, Durey, Satie, Milhaud, Ravel et Debussy. Les critiques soulignent que dans cet alignement, c'est sans doute Rodolphe Mathieu qui propose l'œuvre la plus atonale. Morin est surpris de la bonne réception qu'il reçoit et écrit sur l'évolution du public canadien à son retour à Paris. En 1923, une tournée marque durablement sa carrière, avec Maurice Ravel et le baryton Victor Brault, pour des festivals Ravel, qui les mènent un peu partout en Europe.

En 1925, revenu au Québec pour quelques mois, il reprend l'enseignement en ouvrant deux studios de musique, où étudie entre autres André Laurendeau. Tout en faisant fréquemment la navette entre les deux continents, il enseigne aussi, à partir de 1929, au Conservatoire national de musique aux côtés de Rodolphe Mathieu, Claude Champagne et Albertine Morin-Labrecque. Par ailleurs, son imprésario Bernard Laberge s'étant associé à une imprésario new-yorkaise, Lucy Bogue, leur bureau représente de plus en plus d'artistes et de compositeurs importants, dont Ravel, Honegger et Schmitt. Pour Morin, les grandes tournées se multiplient, particulièrement au Québec, traçant la voie aux Jeunesses musicales qui seront fondées en 1949 .

Morin s'implique alors dans la création de la section montréalaise de Pro Musica ${ }^{2}$, une société new-yorkaise. La section montréalaise attire rapidement les notables et les amis de Morin. Un comité prépare des rapports sur la musique présentée, sur les compositeurs vivant dans la ville, sur les organisations locales, sur les artistes de l'organisation et leurs activités et sur les partitions qui sont conservées par l'organisation. La contrepartie internationale réunit plusieurs des compositeurs les plus importants et leur objectif est de rapprocher compositeurs, musiciens et auditoire. La devise choisie par la section montréalaise est éloquente : «Le tact dans l'audace, c'est de savoir jusqu'où
Morin fera de très nombreux concerts et causeries sous l'égide de Pro Musica. Par exemple, Darius Milhaud et Alfredo Casella viennent y présenter leurs œuvres et Morin donne en 1927 une importante causerie illustrée d'œuvres sur les compositeurs autrichiens et hongrois: il y joue du Schoenberg, du Bartók, du Berg et du Korngold. Autre initiative de Pro Musica, un appel est lancé aux compositeurs canadiens pour un concert au Ritz-Carlton en avril 1927. On y joue Mathieu, Tanguay, O'Brien, Willan et Champagne, mais aussi des œuvres de deux compositrices canadiennes, Corinne Dupuis-Maillet (1895-1989) et Gena Branscombe (1881-1977). Morin en profite pour y faire la création d'une œuvre inspirée de chants «esquimaux» sous le nom de James Callihou, pseudonyme qui restera caché jusqu'après sa mort. Il pousse la mystification jusqu'à présenter une entrevue avec ce compositeur qui serait originaire d'Edmonton et le décrit ainsi: «C'est pour le moins un être exceptionnellement souple, cultivé, aristocratique et détaché des contingences ordinaires ${ }^{3}$. S Sous ce masque, il fera quelques autres compositions, toujours inspiré par les chants traditionnels recueillis par l'ethnologue Marius Barbeau, avec qui il établira des liens suivis.

Malgré ces essais en composition, c'est sa carrière d'interprète qui domine: mentionnons un concert tout Debussy en 1927 avec la soprano Cédia Brault et le violoniste Robert Imandt, un concert avec la célèbre soprano Éva Gauthier (1885-1958) à New York en 1928 dans un répertoire avant-gardiste, sa participation à un concert Ravel à Montréal, dans Ma mère l'Oye, en version piano quatre mains avec le compositeur, un festival de musique canadienne en 1929 à Montréal, où Morin inclut plusieurs de ses œuvres, sous son nom et sous celui de son pseudonyme, James Callihou. En 1929, il doit cependant s'arrêter pour des raisons de santé, une pause qui durera plus d'un an. C'est dans cette période, à partir de 1926, que Léo-Pol Morin commencera à publier régulièrement dans les 
journaux comme critique musical et musicographe, dans La Patrie (1926-1929), La Presse (1929-1931) et Le Canada (1933-1941).

Quand son imprésario Bernard Laberge fait faillite en 1931, Morin se repositionne rapidement en profitant de l'essor rapide de la radio. Entre 1930 et 1941, il participera à 79 émissions en direct, à la station CKAC (ouverte en 1922) et à Radio-Canada (ouverte en 1936). Il y reprend l'ensemble de son répertoire en y ajoutant la mission éducative qu'il a faite sienne. Par ailleurs, il continue à offrir des concerts de musique de chambre en salle et ses concerts-causeries obtiennent de grands succès. Quand, en 1934, la Société des concerts symphoniques de Montréal ${ }^{4}$ voit le jour, Léo-Pol Morin est soliste au concert inaugural avec le Caprice brillant pour piano et orchestre de Mendelssohn. Il est réinvité en 1935 et en 1936 . De janvier 1936 à son décès en 1941, il prend la relève d'Alfred Laliberté à l'École supérieure d'Outremont, tout en gardant des élèves en privé. Il est reconnu excellent professeur de piano, ainsi trois de ses élèves se présentent au Prix d'Europe en 1939 dont Jean Papineau-Couture, Lorraine Gaboury et Paule-Aimée Bailly qui remporte le prix. Pendant les dernières années de sa vie, Morin ne joue qu'à la radio et c'est en se déplaçant avec une équipe de la radio que l'accident survient. Il a alors 48 ans.
Plusieurs organismes lui rendent hommage, pour sa carrière d'interprète, de critique et d'enseignant.

Claudine Caron a su mettre en lumière, en suivant la trace de ses concerts, la modernité de Léo-Pol Morin. Ce sont les moments les plus intéressants de son livre. Chaque chapitre se clôt sur une conclusion réflexive où elle plaque des concepts théoriques pour soutenir son analyse - Hans Robert Jauss, Georges Leroux, Lydia Goehr, Ernst Bloch ou Marcel Fournier -, mais, si ces recours sont certainement nécessaires dans une thèse de doctorat, ils sont ici maladroits, insuffisamment développés pour servir efficacement son propos. On peut aussi regretter une biographie plus complète de Léo-Pol Morin, où tous les aspects de sa vie seront mieux intégrés. Malgré ces réserves, il faut saluer cette publication éclairante sur un précurseur important de la modernité au Québec.

1. Claudine Caron est docteure en musicologie, spécialisée en histoire de la musique du Québec et du Canada. Elle est membre du Centre de recherche interuniversitaire sur la littérature et la culture québécoise (CRILCQ).

2. Une organisation différente de la société Pro Musica, encore active à Montréal.

3. Léo-Paul Morin (1929), "Une heure avec le compositeur James Callihou ", La Patrie, 19 mars.

4. Organisme qui prendra le nom d'Orchestre symphonique de Montréal en 1953. 